\title{
Two-electron singlet states in semiconductor quantum dots with Gaussian confinement: a single-parameter variational calculation
}

\author{
Bahadir Boyacioglu $^{1}$, Mesude Saglam ${ }^{2}$ and Ashok Chatterjee Ch,4 $^{3,4}$ \\ ${ }^{1}$ Vocational School of Health, Ankara University, 06290 Kecioren, Ankara, Turkey \\ ${ }^{2}$ Department of Physics, Faculty of Sciences, Ankara University, 06100 Tandogan-Ankara, Turkey \\ ${ }^{3}$ Department of Physics, Bilkent University, 06800 Bilkent, Ankara, Turkey
}

Received 24 July 2007, in final form 25 September 2007

Published 17 October 2007

Online at stacks.iop.org/JPhysCM/19/456217

\begin{abstract}
The problem of two electrons in a three-dimensional quantum dot with Gaussian confinement is investigated for the singlet pairing by a variational method with a very simple wavefunction containing only a single parameter and a Jastrow-like factor, which is shown to yield fairly good results for deep confining potentials. The calculation is also performed for a few realistic semiconductor quantum dots and the phase diagrams for the two-electron singlet states are obtained for these materials. The pair density function is calculated for several parameter values and its peak positions are obtained as a function of the confinement length and the depth of the potential to study the behaviour of the electron-pair size. The size of the bound pair of electrons is also obtained by directly calculating the average distance between the two electrons in three different ways and compared with the pair correlation results. It is furthermore shown that, other properties remaining the same, the two-electron energy and the electron-pair size depend crucially on the effective electronic mass and the dielectric constant of the material. Finally, the ways of improving the wavefunction are also indicated.
\end{abstract}

Interest in the subject of quantum dots has continued unabated in the last two decades or so for several reasons (see [1] for review). First of all, it has an intrinsic appeal from the point of view of fundamental physics because the natural length scales (confinement lengths, donor Bohr radius, exciton radius) involved in it are of the order of a few nanometres, where the quantum effects are expected to show up in their full glory. In fact, quantum dots can be considered as ideal quantum systems containing a discrete but variable number of electrons resembling giant artificial atoms, which can provide a tiny laboratory where the predictions of quantum mechanics can be tested rigorously. Also, the electron-electron energy plays a very

4 On leave from: School of Physics, University of Hyderabad, Hyderabad, India. 
important role in quantum dots, in general, because its energy scale is of the same order as that of the confining potential. Of course there are a few other competing energy scales as well in these systems and that makes the perturbative treatments for these systems quite inappropriate. Because of the nanoscale extensions in all the directions of space, the quantum dots possess discrete energy levels that can be tuned at will, and consequently these structures exhibit very many new physical phenomena that are quite different from those of their bulk counterparts and are exclusively of quantum origin, like charge quantization and Coulomb blockade, which give rise to the possibility of realizing novel devices like single-electron transistors, quantum dot lasers and ultrafast computers. One of the important technological advantages with the quantum dots is that these structures can be fabricated in different shapes and sizes. It turns out that the nature and details of the confining potential depend to a large extent on the geometry of the quantum dot and the external voltage applied to the micro-electrodes and thus can be changed at will, which provides a unique opportunity of getting the electron energy spectra with the desired properties. The reduced dimensionality with enormous design flexibility, the finite particle number and the presence of several comparable length and energy scales have indeed made this new research area of mesoscopic systems extremely fascinating, with lots of challenges, and opened up a new frontier in condensed matter physics with tremendous potentiality to revolutionize modern technology.

One of the major issues in the theoretical simulation of a quantum dot is to provide a prescription for the attractive confining potential. In the simplest approach, one takes the electron to be confined in an infinite potential well. This model is however not very realistic since the force experienced by the electron within the dot is not zero in reality. Sikorski and Merkt [2] have found experimentally that the resonance frequencies of quantum dots do not depend on the number of electrons in the quantum dot, which indicates that the excitation spectrum of a quantum dot is not influenced by the electron-electron interaction. Later, Meurer et al [3] studied the few-electron quantum dot energy spectrum using the far-infrared spectroscopy on GaAs quantum dot and have observed that resonance frequencies have only a weak dependence on the electron number. Independence of the dipole transitions from the electron number was also approximately observed in GaAs-GaAlAs quantum dots by Ashoori et al [4] and in the InGaAs-GaAs-AlAs system by Drexler et al [5]. All these results suggest an interesting feature about the quantum dot. It is known from Kohn's theorem [6] that the cyclotron frequency of a translationally invariant electron system is independent of the electron density and of the form of the electron-electron interaction. This theorem is a consequence of the fact that the electric dipole of the radiation couples only to the centre of mass of the electrons and does not affect the relative motion. Peeters [7] has shown that the position of the resonance lines in the magneto-optical absorption of a quantum dot with a parabolic confinement potential is also independent of the electron-electron interaction and the number of electrons in a quantum dot. Similar results have also been obtained by Yip [8] and Li et al [9]. These results indicate the validity of the so called generalized Kohn's theorem in a quantum dot, according to which one can conclude that the bare confining potentials are almost parabolic in these systems. Consequently, a large number of theoretical investigations have been carried out in this area in the last two decades, taking the confinement potential as parabolic [10].

However, in recent years a few groups [11] have shown that their experimental results do not agree with the generalized Kohn theorem. Their observations reveal that the confining potential should be nonparabolic and should have the shape of a finite potential well. Szafran et al [12] and Bednarek et al [13] have been able to qualitatively describe the charging of quantum dots using a three-dimensional spherical rectangular potential well of finite depth. Szafran et al [14] have quantitatively explained the capacitance-spectroscopy data for self-assembled quantum dots using a three-dimensional cylindrical potential well. 
Recently, Adamowsky et al [15] have proposed a Gaussian attractive confining potential for the investigation of the properties of excess electrons in quantum dots. This potential has a finite depth and in the neighbourhood of the dot centre would behave like a parabolic potential and would also approximately satisfy the generalized Kohn theorem. Furthermore, in contrast to the rectangular potential well, it is continuous at the dot boundaries, and this makes it easier to handle mathematically. Also, it has a central minimum as required for a physical potential, and the force experienced by the particles within this potential well is nonzero, which is also a desirable feature. The other advantages with the Gaussian confining potential vis$\grave{a}$-vis a parabolic potential are that the former allows for, in addition to the excitations, the ionization and tunnelling processes. We would like to mention in passing that the Gaussian potential has proved to be a useful potential in various branches of physics and has been solved approximately for a single-particle problem by several authors [16].

Adamowsky et al [15] have used a variational method to study the bound state problem of a pair of electrons in a quantum dot with Gaussian confinement in two and three dimensions for singlet and triplet pairing and have shown that singlet pairing has the lower energy. A number of variational approaches [17] in general have been used in the literature to account for both single- and two-electron systems in quantum dots with various kinds of confining potentials. In the present paper we shall also use a variational method and confine our discussion to the three-dimensional case and the singlet pairing. Adamowsky et al [15] have chosen a wavefunction (hereafter referred to as the ASSB wavefunction) that has the proper symmetry as required by the Pauli principle and also contains a Coulomb correlation factor which is an exponential function of the electron-electron separation. They have made a calculation with about 200 parameters to obtain the required accuracy. But their wavefunction suffers from a serious lacuna. For $r_{12}$ equal to zero, their wavefunction still survives instead of collapsing, which implies that the wavefunction allows the two particles to occupy the same position in the configuration space, in clear violation of the Coulomb correlation. Thus, though the ASSB wavefunction satisfies the Pauli correlation, it does not satisfy the Coulomb correlation properly. In the present paper, our aim is to introduce a Coulomb correlation factor in the wavefunction that would take care of both the long- and the short-range correlations for this problem. Furthermore, we will choose a very simple wavefunction containing only a single variational parameter and we will show that this simple wavefunction may be considered very good for a quantum dot for a certain range of the confining potential, and reasonably good for a broad range of the confinement length. We shall also indicate the ways of improving the wavefunction so that one can get results of desired accuracy over the entire range of the confinement length.

The Hamiltonian of a system of two electrons moving in a three-dimensional quantum dot can be written as

$$
\mathcal{H}=-\frac{\hbar^{2}}{2 m^{*}} \sum_{i=1}^{2} \nabla_{\vec{r}_{i}}^{2}+\sum_{i} V\left(r_{i}\right)+\frac{\beta}{\left|\vec{r}_{1}-\vec{r}_{2}\right|},
$$

where $\vec{r}_{i}(x, y, z)$ is the position coordinate of the $i$ th particle, $m^{*}$ is the effective electron mass, $\beta$ is the strength of the Coulomb repulsion between the two electrons and is given by $e^{2} / 4 \pi \varepsilon_{0} \varepsilon$, where $\varepsilon_{0}$ is the permittivity of the vacuum and $\varepsilon$ is the dielectric constant of the medium, and $V\left(r_{i}\right)$ is the confining potential for the $i$ th electron and is chosen following [15] as

$$
V\left(r_{i}\right)=-V_{0} \mathrm{e}^{-r_{i}{ }^{2} / 2 R^{2}},
$$

where $V_{0}$ denotes the depth of the potential and $R$ gives a measure of the range of the potential and thus represents the effective confinement length or the size of the quantum dot. It may be noted that the choice of semiconductor host material permits us to vary the effective electron 
mass (because of different band structures) and the screening of the repulsive electron-electron interaction via the effective dielectric constant. Depending on the material, $m^{*} / m_{0}$ (where $m_{0}$ is the bare electron mass) can vary over a wide interval from 0.05 to 1.5 . Retaining up to the quadratic term after expanding the potential (2) for $r \ll R$ yields the usual parabolic potential

$$
V(r)=-V_{0}+\gamma^{2} r^{2}
$$

where $\gamma^{2}=\left(V_{0} / 2 R^{2}\right)$. The corresponding Schrödinger equation for (3) is exactly soluble, with the following energy spectrum in three dimensions:

$$
E_{n l}=-V_{0}+\gamma(4 n+2 l+3),
$$

where $n, l=0, \pm 1, \ldots$

For the sake of comparison we shall first consider the single-particle problem and obtain a few low-lying energy levels for the Gaussian potential (2) using the Ritz variational method. For the states $(n=0, l=0),(n=0, l=1),(n=1, l=0)$, and $(n=0, l=2)$, we choose the following trial wavefunctions:

$$
\begin{aligned}
& \psi_{00}(\vec{r})=\left(\frac{2 \alpha}{\pi}\right)^{3 / 4} \mathrm{e}^{-\alpha r^{2}} \\
& \psi_{01}(\vec{r})=\left(\frac{128 \alpha^{5}}{9 \pi}\right)^{1 / 4} r \mathrm{e}^{-\alpha r^{2}} \\
& \psi_{10}(\vec{r})=\left(\frac{2 \alpha^{3}}{3 \pi^{3 / 2}}\right)^{1 / 2}\left(-\frac{3}{2}+\alpha^{2} r^{2}\right) \mathrm{e}^{-\frac{1}{2} \alpha^{2} r^{2}} \\
& \psi_{02}(\vec{r})=\left(\frac{\alpha^{7}}{3 \pi^{3 / 2}}\right)^{1 / 2} r^{2} \mathrm{e}^{-\frac{1}{2} \alpha^{2} r^{2}} Y_{2}^{0}(\theta, \phi),
\end{aligned}
$$

where $Y_{2}^{0}(\theta, \phi)$ is the spherical harmonics and $\alpha$ is a variational parameter. For a singleparticle parabolic potential, $\alpha^{-\frac{1}{2}}$ physically gives the length scale over which the ground state wavefunction dies out. So it gives a signature of the bound state. Obviously a larger value of $\alpha$ leads to a stronger single-particle binding. It is well known that if one chooses the wavefunctions (5)-(8) as trial wavefunctions for a single-particle parabolic potential and performs variational calculations, one would get exact results for $\alpha$ and the energies. However, for a Gaussian potential, the variationally obtained values of $\alpha$ will actually lead to approximate wavefunctions and yield upper bounds to the exact energies. But, if the size of the quantum dot is not too large, the Gaussian potential may be pretty close to the parabolic potential for all practical purposes and then $\alpha$ will be a reasonably good parameter to describe the wavefunction of the Gaussian potential. This is what we will really show in figure 1 . Choosing the same units as in [15], i.e. the donor Bohr radius $\left(a_{\mathrm{D}}\right)$ as the unit of length and the donor Rydberg $\left(R_{\mathrm{D}}\right)$ as the unit of energy, we obtain the variational upper bounds to the energy of the states described by functions (5)-(8) for $V_{0}=50 R_{\mathrm{D}}$ and $R=a_{\mathrm{D}}$. The values of $a_{\mathrm{D}}$ and $R_{\mathrm{D}}$ are chosen as $10 \mathrm{~nm}$ and $6 \mathrm{meV}$ respectively, which correspond to a $\mathrm{GaAs}$ quantum dot in the $\mathrm{Al}_{0.3} \mathrm{Ga}_{0.7} \mathrm{As}$ matrix. The results are shown in figure 1, where we have also shown the exact results for the corresponding states of the parabolic potential for the sake of comparison. We have also shown for the Gaussian potential the variational results of Adamowski et al [15]. One can clearly see that our one-parameter results are pretty good as compared to those of [15], which involves a large number of variational parameters. This implies that, at least for a single-particle problem of a Gaussian potential, the decay of the wavefunctions can still be reasonably described by a Gaussian functions with a single parameter $\alpha$. Also, our results are in good agreement with the large- $N$ expansion results of Chatterjee [16], which gives the lower bound, in contrast to the present variational results, which provide the upper bound. In fact, these two results can 


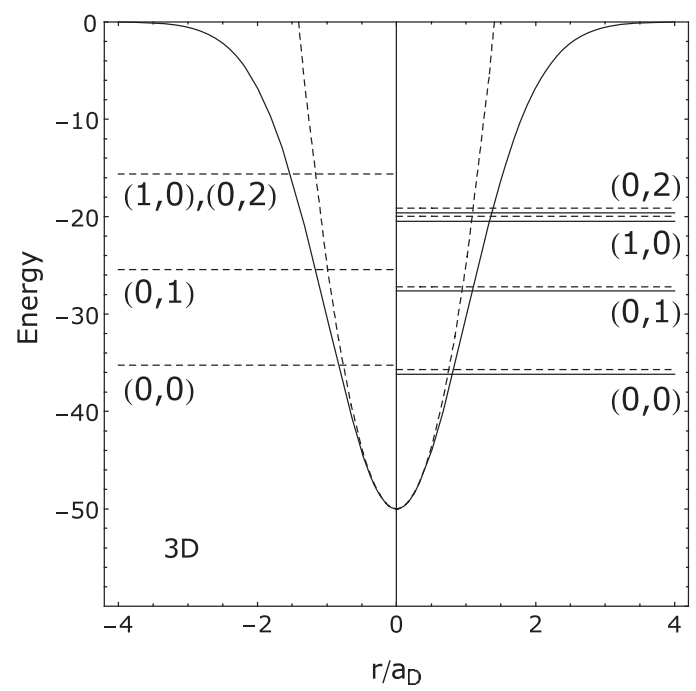

Figure 1. One-electron energy levels for 3D Gaussian (solid lines) and harmonic-oscillator (dashed lines on the left side) potentials with $V_{0}=50 R_{\mathrm{D}}$ and $R=a_{\mathrm{D}}$. The energy levels are labelled by quantum numbers $(n, l)$. The dashed lines on the right side show the results of Adamowsky et al [15] for the Gaussian potential. Energy is expressed in units of $R_{\mathrm{D}}$ and length in $a_{\mathrm{D}}$. The shapes of both the potential energies $\left(V / R_{\mathrm{D}}\right)$ are also shown as functions of the distance $\left(r / a_{\mathrm{D}}\right)$ from the quantum dot centre. The confining potential curves are as usual independent of materials.

help us to further narrow down the bounds within which the exact results lie. However, the important point here, as has been rightly mentioned by Adamowski et al [15], is that except for the ground state the parabolic potential cannot be taken as a good approximation of a Gaussian potential because of the large differences of the latter's energy levels from those of the parabolic potential. In fact, the deviation becomes larger and larger as one considers higher and higher excited states. Furthermore, not less importantly, the Gaussian potential also has a few qualitative differences from the parabolic potential. For instance, it has certain degeneracies which are absent in the parabolic potential spectrum and therefore can afford to display a much richer variety of physical phenomena than a parabolic potential. In what follows, we shall consider the problem of a two-particle system in a Gaussian quantum dot, which is the main subject matter of this paper.

As we have already pointed out, the ASSB wavefunction has a serious drawback, for it allows the two electrons to occupy the same position in the configuration space, which is not permitted by Coulomb repulsion. Thus, although the ASSB wavefunction incorporates the effect of Coulomb repulsion at large distances, it lacks the short-range Coulomb correlation. Another point is that their wavefunction contains around 200 parameters and thus involves a highly intensive numerical computation. The main goal of the present paper is to introduce a simple one-parameter wavefunction that, we will show, works pretty well, maybe in a certain range. We write the two-particle wavefunction for the singlet pairing as

$\psi\left(\vec{r}_{1}, \vec{r}_{2}\right)=\prod f\left(\vec{r}_{i}-\vec{r}_{j}\right) \operatorname{det}\left(\psi_{i}\left(r_{j}\right)\right)=\left[\frac{4 \alpha^{2}}{\sqrt{3} \pi^{3 / 2}}\right] f\left(\vec{r}_{1}-\vec{r}_{2}\right) \mathrm{e}^{-\alpha\left(r_{1}{ }^{2}+r_{2}{ }^{2}\right)}$,

where $f\left(\overrightarrow{r_{1}}-\overrightarrow{r_{2}}\right)$ is the Jastrow-type Coulomb correlation factor (Jastrow pre-factor), which also takes care of the fact that two electrons are not allowed to occupy the same position. For simplicity we take it as $f\left(\vec{r}_{1}-\overrightarrow{r_{2}}\right) \simeq\left|\overrightarrow{r_{1}}-\overrightarrow{r_{2}}\right|=r_{12}$. To our knowledge, the Jastrow correlation factor was first introduced in the context of the helium problem. It has also been 


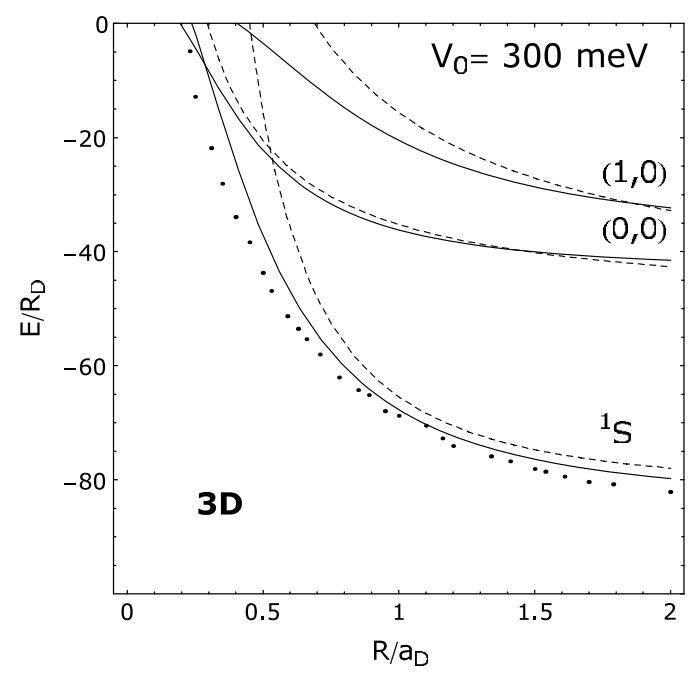

Figure 2. Energy levels of the singlet state of two electrons confined in Gaussian (solid curves) and harmonic-oscillator (dashed curve) potentials as functions of the dimensionless effective confinement length $R / a_{\mathrm{D}}$ for $V_{0}=50 R_{\mathrm{D}}$. The dots refer to the result of Adamowsky et al [15]. One-electron levels $(0,0)$ and $(1,0)$ are also shown for the potentials using our wavefunctions (5) and (7) respectively. The units are same as in figure 1.

used in the wavefunction for the quantum Hall effect [18]. In the polaron problem, or more precisely in the bulk bipolaron problem, it was first used by Chatterjee et al [19] and was shown to incorporate the Coulomb correlation effect pretty efficiently. In a parabolic quantum dot, it was first used by Mukhopadhyay and Chatterjee [20] for the bipolaron problem. The Jastrow correlation factor has also been used in quantum dots by several other authors [21]. It has been shown by Ciftja and Faruk [21] that the Jastrow factor takes care of the Coulomb correlation effect quite efficiently and compares well with the results of exact diagonalization and captures the essential features of a correlated system. Ciftja and Anil Kumar [21] gave a very useful discussion of the variational method using the Jastrow method. One may also note that in addition to the Jastrow factor the wavefunction (9) contains a product of the singleparticle Gaussian functions with a single variational parameter $\alpha$, since there is no reason to make any distinction between the two electrons, which implies that we have assumed that even for the composite two-particle system the bound state will be described by a single parameter. Of course one can introduce more parameters in the wavefunction, and we believe this may improve the results a little bit quantitatively. It is evident that wavefunction (9) is clearly symmetric in the position coordinates $\vec{r}_{1}$ and $\overrightarrow{r_{2}}$ and therefore it describes the right statistics. In our opinion, (9) is the simplest possible wavefunction with all the desired properties for the present problem. It seems useful at this stage to perform the following transformations:

$$
\overrightarrow{r_{1}}=\frac{[\vec{v}+\vec{u}]}{\sqrt{2}} ; \quad \overrightarrow{r_{2}}=\frac{[\vec{v}-\vec{u}]}{\sqrt{2}} .
$$

In terms of $\vec{u}$ and $\vec{v}$, both the Hamiltonian and the wavefunctions look simpler and the calculation of the energy expectation values becomes a lot more easier. Finally, however, the integrations and minimizations are done numerically. We obtain the energy values in units of $R_{\mathrm{D}}$ for both the parabolic potential (3) and the Gaussian potential (2) for singlet states in three dimensions as a function of the dimensionless effective confinement length $R / a_{\mathrm{D}}$ for the same GaAs quantum dot system as mentioned above. The results are shown in figure 2, 
where we have also plotted the ASSB results for comparison. We have furthermore shown the single-particle results for both the parabolic and the Gaussian potential obtained with our wavefunctions (5) and (7). It is worthwhile to point out that for large confinement lengths $\left(R \gg a_{\mathrm{D}}\right)$ the parabolic potential can be considered a good enough approximation of the Gaussian potential for the single-particle problem. This however is not so true for the twoparticle problem. For the higher excited states, in fact, the discrepancy is even more, as also shown in [15]. The reason is of course understandable. For small confinement lengths, even for the single-particle problem, the parabolic potential results are not so good and become still worse for the excited states as compared to the Gaussian potential results. The reason is of course not difficult to understand. Since for small confinement lengths $\gamma$ is large, equation (3) is not a good approximation of the Gaussian potential (2), and therefore one cannot expect parabolic potential results to compare well with the Gaussian potential results for small $R$. The deviation is more glaring for the two-particle problem. Comparison of our two-particle results with those of [15] shows that for intermediate range of the confinement length the results agree pretty well, but for large and small confinement lengths the results of Adamowsky et al [15] are slightly better than ours. But in view of the extreme simplicity of our single-parameter wavefunction vis-à-vis the huge ASSB wavefunction, our results may be considered pretty good.

Since the Gaussian potential (2) has a finite depth, it can naturally accommodate only a finite number of bound states for a single particle [16]. For the definition of binding, we follow the prescription of Adamowsky et al [15], which for a two-particle system is given by $E_{s}(2)<E_{0}(1)$, where $E_{s}(2)$ is the two-particle ground state (GS) energy in the singlet state and $E_{0}(1)$ is the single-electron GS energy. According to this definition the unbound phase consists of one electron being inside the quantum dot with the other being outside. For a single-particle system, the binding is given by the condition $E_{0}(1)<0$. One can observe from figure 2 that there exists a critical value of the confinement length below which the two-particle singlet state becomes unbound. Thus we can easily obtain a phase diagram for the two-particle states for a Gaussian quantum dot. In figure 3 we plot this phase diagram. The solid curve refers to our result and implies that the parameter values above this curve correspond to the phase in which the two-particle bound state is the ground state, and below this curve the unbound state is a more favourable phase. Thus the solid curve gives the critical parameter values at which an electron can tunnel out of the Gaussian quantum dot. The bound state to unbound state crossover can be understood in a simple way. When the quantum dot is small, according to the uncertainty principle the kinetic energies of the electrons should be large and also the average Coulomb correlation would be pretty large for the narrow range of the confining potential, and in this case the positive energy coming from the kinetic and the Coulomb energies is too large to be compensated for by an attractive confining potential of small strength $V_{0}$. Thus in such a case one needs a pretty strong confining potential to have a bound state of two electrons. It seems that our calculation agrees fairly well with those of Adamowsky et al for small confinement lengths or more precisely for deep potential wells. Below a certain value of the depth of the confining potential, there is however a large discrepancy between our results and those of [15]. According to our results, the potential required to provide a bound state seems to saturate to a constant value, which means that above a certain confinement length a minimum critical value of $V_{0}$ is required to provide a bound state, and this $V_{0}$ turns out to be independent of the size in the bulk limit. This result is a little unrealistic from physical grounds. For a single particle, the BohrSommerfeld quantization condition or the WKB approximation for the GS energy dictates that the minimum value of $V_{0}$ to sustain a bound state in a Gaussian potential is proportional to $1 / R^{2}$. Thus one will always have a single-particle bound state in a Gaussian potential for any finite value of $V_{0}$. For a two-particle system one can understand the situation in a qualitative 


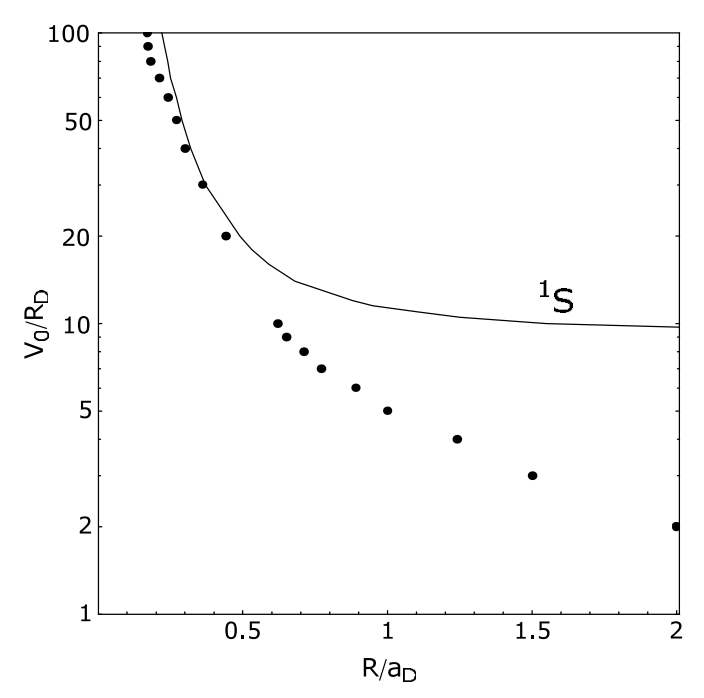

Figure 3. Phase diagram in the $R-V_{0}$ plane for the two-electron system in the Gaussian potential in a GaAs quantum dot mentioned in the text. The solid line refers to the critical values of the quantum dot parameters above which the two-electron state is a bound state. The parameters on the curve give values for which an electron can tunnel through the quantum dot. The solid dots refer to the results of [15].

way. For example, one would expect that at large confining lengths the kinetic energies of the two electrons would be very small according to the uncertainty principle, and the electronelectron interaction energy would also be very small because of the Coulomb correlation, and then even a small confining potential should give a bound state. This is what has been found by Adamowsky et al. The lacuna in our results for a shallow potential (at small values of $V_{0}$ ) may be attributed to the simplistic nature of the trial wavefunction we have chosen, and the plateau in our phase diagram thus seems to be an artefact of our approximation. Thus it appears that a single-parameter Gaussian wavefunction may not be a good enough wavefunction for a shallow Gaussian potential, or in other words our simplistic treatment does not give a proper bulk limit.

We have calculated the singlet state energy values of a two-particle system in a few real quantum dots of semiconducting materials such as GaAs, GaSb, InAs and InSb using the same Gaussian confining potential. The results are shown in figure 4. The results indicate that GaAs supports the lowest GS energy and the GS energy value is highest in InSb among the materials considered in the present work. One probable reason for this is that, other properties remaining more or less the same, the smaller the effective electron mass, the larger the kinetic energy. Thus the associated quantum fluctuations increase with decreasing effective electron mass, leading to a higher energy. Therefore, the electron effective mass turns out to be a crucial parameter in the energetics of a quantum dot. However, it must be mentioned here that the materials considered in this paper have a similar range of values for the dielectric constant. A much higher value of the dielectric constant should reduce the Coulomb repulsion considerably and consequently lower the energy. Thus one would expect that two independent effects having their genesis in the electron effective mass and the dielectric constant would essentially decide the nature of delocalization depending on their relative strengths and will eventually compete with the confining potential to give the GS energy for a two-particle system in a quantum dot. Next we calculate the energy values of the two-particle system for singlet pairing as a function of the depth of the attractive Gaussian confining potential for the semiconductor 


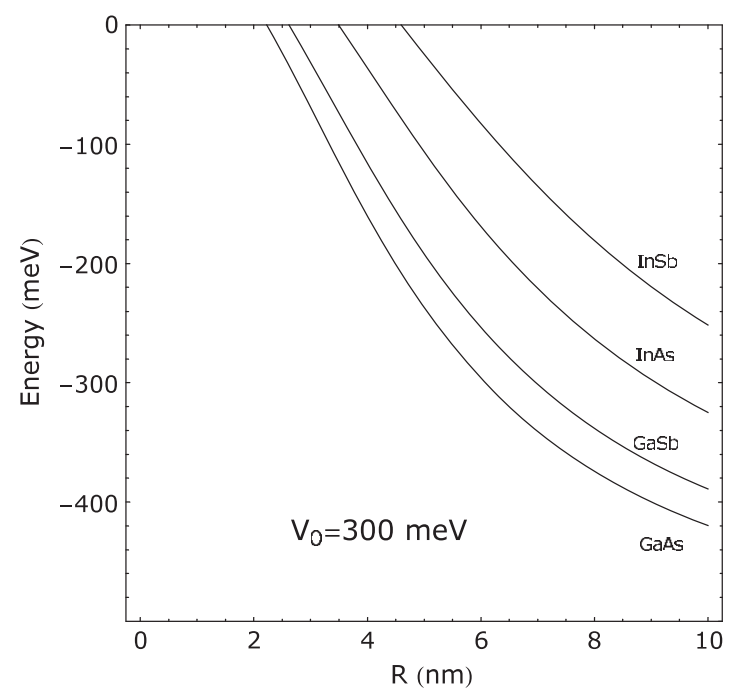

Figure 4. Energy levels of the two-electron singlet states in a few realistic Gaussian quantum dots as a function of the effective confinement length for $V_{0}=300 \mathrm{meV}$. The effective electronic masses in GaAs, GaSb, InAs and InSb are given respectively by $6.012 \times 10^{-32}, 4.28 \times 10^{-32}, 2.37 \times 10^{-32}$ and $1.37 \times 10^{-32} \mathrm{~kg}$.

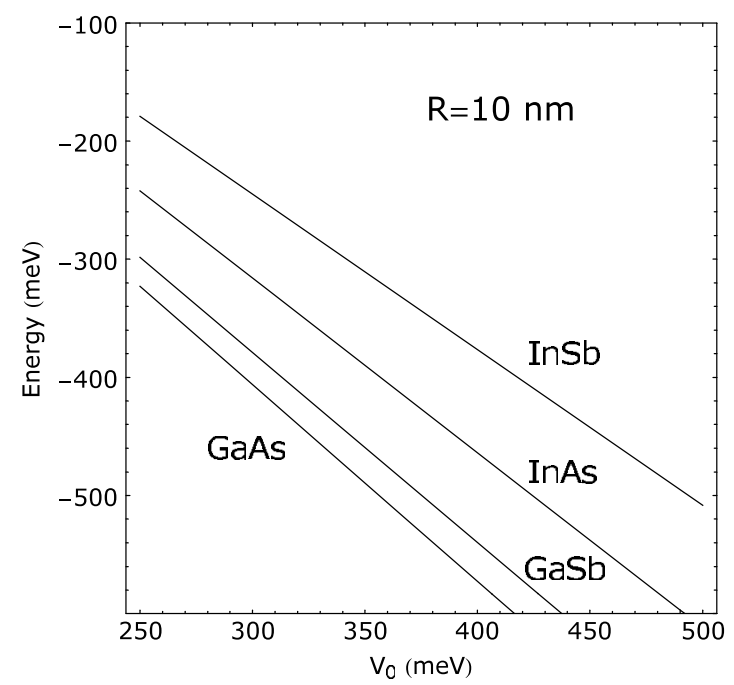

Figure 5. Energy levels of the two-electron singlet states in a few Gaussian quantum dots as a function of the depth of the confining potential for $R=10 \mathrm{~nm}$.

quantum dots considered above for a fixed value of the confinement length $(R=10 \mathrm{~nm})$. The results are shown in figure 5. One can clearly see that as the depth of the potential increases the energy of the two-particle state becomes lower and lower, almost in a linear fashion. This is of course along the expected line, because as the potential minimum becomes lower and lower the energies would naturally come down more and more and the system would accommodate a larger number of bound states. Again, as expected from other curves, the curve for GaAs lies lowest and that for InSb highest. From figure 4 and the single-particle GS results in the 


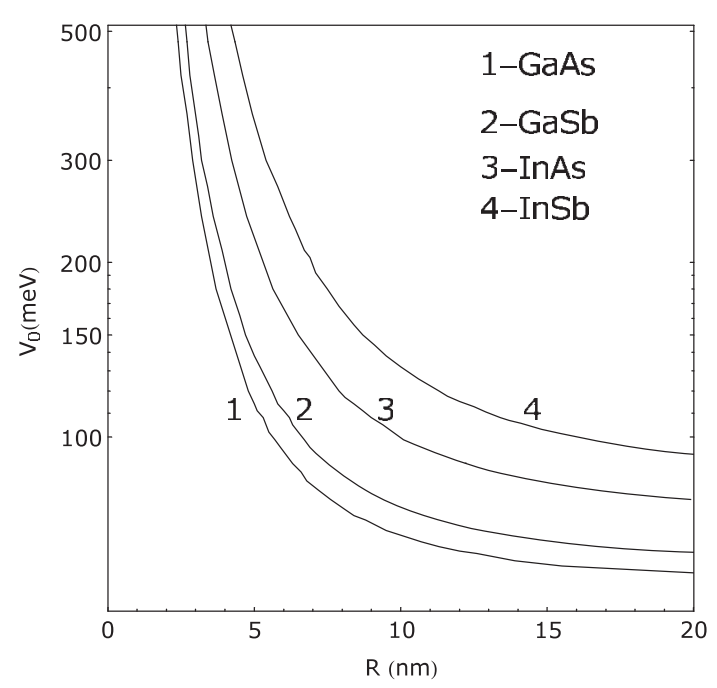

Figure 6. Phase diagrams for the two-electron singlet states in a few realistic Gaussian quantum dots.

same materials we can determine the phase diagrams for the two-electron singlet bound states in GaAs, GaSb, InAs and InSb quantum dots as a function of the effective confinement length. The results are shown in figure 6. It is evident that the bound phase is widest in the GaAs dot while in InSb it is smallest. Thus we find that the conclusion about the strength of binding in these materials can be essentially predicted from the energy curves. This may however be fortuitous. Our results however do not give the correct phase diagram below a certain value of $V_{0}$. In fact, below this value of $V_{0}$ we get plateaus instead of a monotonic decrease in $V_{0}$. This behaviour, as we have already mentioned, is an artefact of our approximation. But it is undoubtedly clear that for $R=10 \mathrm{~nm}, V_{0}>150 \mathrm{meV}$ approximately will give singlet bound states in all the materials considered here.

It would be interesting to determine the pair density function and study how it varies with the range and depth of the confining potential. The pair density function $I(\vec{r})$ can be most effectively represented in terms of the relative coordinates, $\vec{r}_{12}$, as follows:

$$
I(\vec{r})=\left\langle\Psi\left|\sum_{i>j} \delta\left(\vec{r}-\vec{r}_{i}+\vec{r}_{j}\right)\right| \Psi\right\rangle
$$

Substituting the wavefunction (9) in (11), we get after some simple algebra

$$
I(\vec{r})=\left(\frac{2048 \alpha^{5}}{9 \pi}\right)^{1 / 2} r^{4} \mathrm{e}^{-2 \alpha r^{2}}
$$

In figure 7 we plot $I(\vec{r})$ as a function of $r$ for several values of $R$ for $V_{0}=300 \mathrm{meV}$. In figure 8 we plot $I(\vec{r})$ as a function of $r$ for several values of $V_{0}$ for $R=10 \mathrm{~nm}$. Figures 7 and 8 show that the pair density peaks at some value of the length $r$, and this value at which the pair correlation is maximum should correspond to the expected size of the electron pair for the parameters chosen. Figure 7 shows that as the range of the confining potential decreases, the electron-electron separation in the singlet state also decreases. Interestingly, however, one can see from the figure that for $R=3 \mathrm{~nm}$ the peak appears at a much higher value as compared to the range of the potential. We can find from figure 3 that for $R=3 \mathrm{~nm}$ the minimum strength of the confining potential required to provide a two-electron bound state is larger than $300 \mathrm{meV}$ and therefore one does not expect a bound state for this case. Since the present peak 


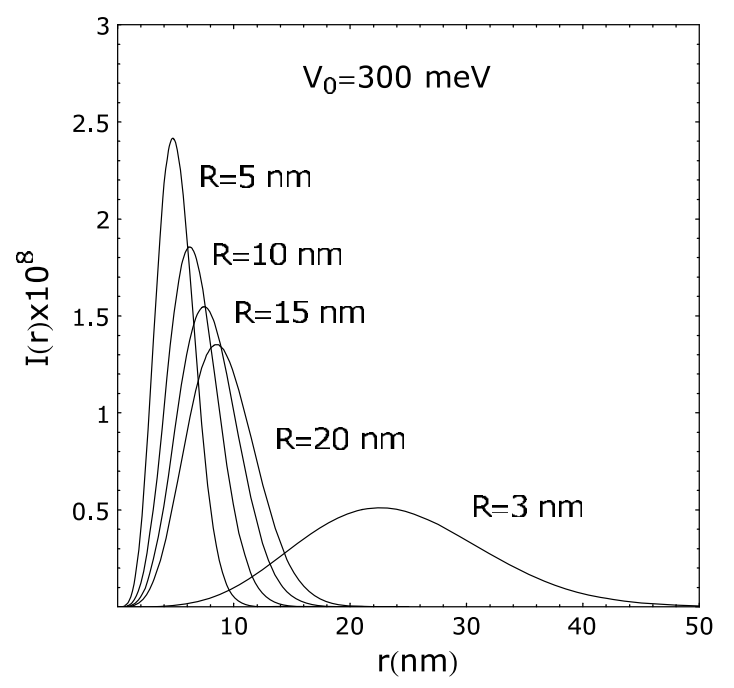

Figure 7. The pair density function as a function of the electron-electron separation for various values of $R$ for $V_{0}=300 \mathrm{meV}$.

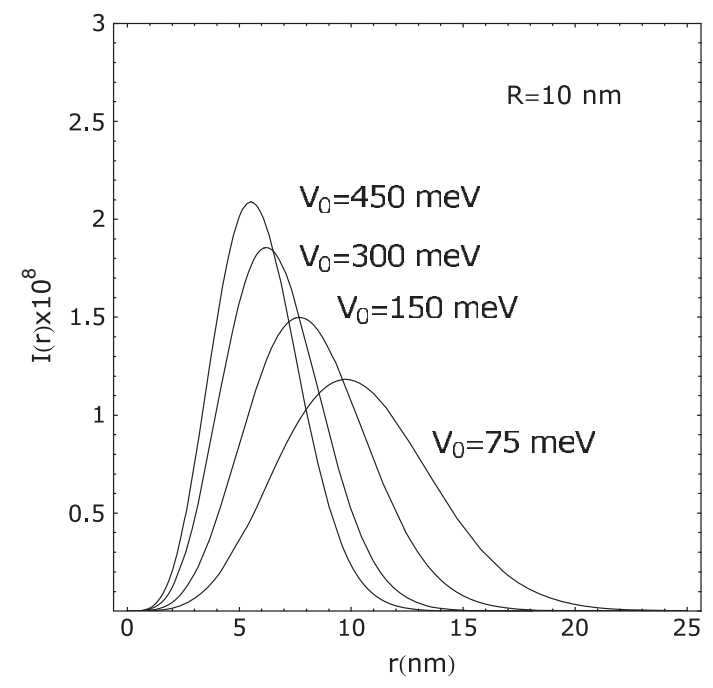

Figure 8. The pair density function as a function of the electron-electron separation for several values of $V_{0}$ for $R=10 \mathrm{~nm}$.

occurs above the zero of the potential, we suggest that it probably corresponds to one of the resonances. This particular aspect requires a more thorough and critical investigation.

In figure 9 we plot the peak position $r_{\text {peak }}$ as a function of $R$ for $V_{0}=300 \mathrm{meV}$ and in figure 10 we show the variation of $r_{\text {peak }}$ as a function of $V_{0}$ for $R=10 \mathrm{~nm}$. It is evident that for a fixed $V_{0}, r_{\text {peak }}$ increases with the range of the potential, while for a given $R, r_{\text {peak }}$ decreases with $V_{0}$. This is understandable because as the range of the confining potential increases the electron separation increases to lower the Coulomb repulsion energy, while with the increase in $V_{0}$ the electron states form at lower energies and thus the electron separation decreases to lower the confinement energy. However, as the strength of the confining potential exceeds a certain 


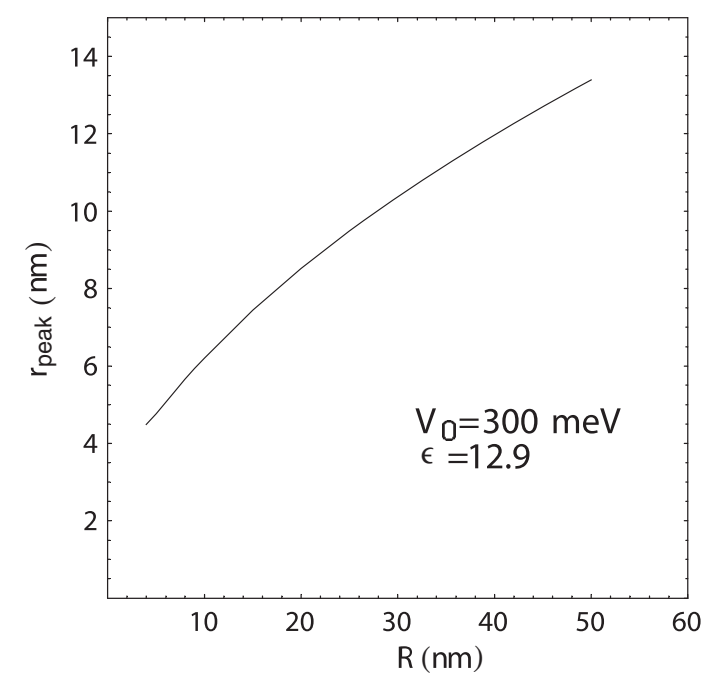

Figure 9. Variation of $r$ corresponding to the peak positions in the pair density function as a function of the effective confinement length $R$ for the depth of the potential, $V_{0}=300 \mathrm{meV}$ and $\varepsilon=12.9$.

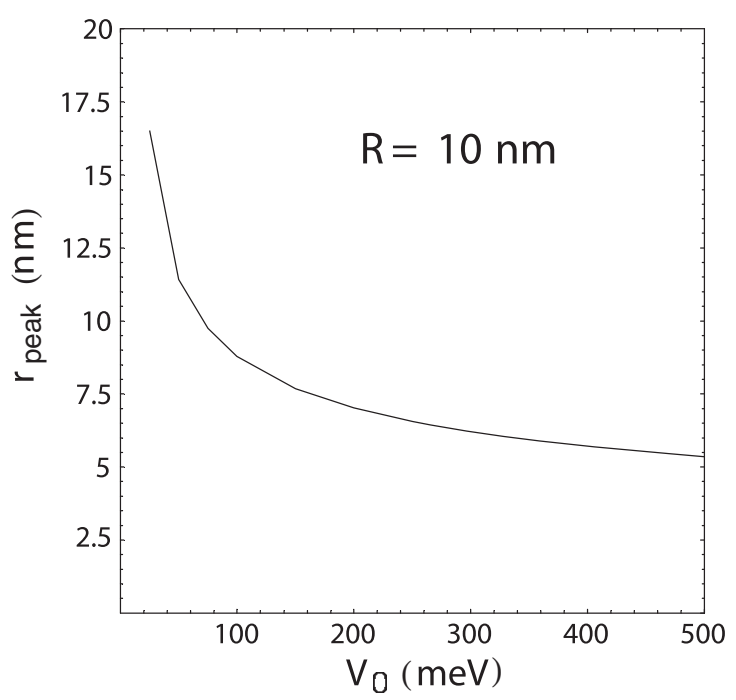

Figure 10. Variation of $r$ corresponding to the peak positions in the pair density function as a function of the depth of the confining potential, $V_{0}$, for $R=10 \mathrm{~nm}$.

limiting value, $r_{\text {peak }}$ saturates to a constant, implying that the electron-electron separation does not decrease below this value. At this value of $V_{0}$, the Gaussian potential essentially looks like an infinitely deep spherical potential well and hence the electron-pair size saturates to a constant in this regime. It is well known that there is no unique way to obtain the size of the electron pair. So it would also be interesting to determine the electron separation by calculating the average quantities like $\left\langle r_{12}\right\rangle,\left\langle 1 / r_{12}\right\rangle^{-1}$ and $\sqrt{\left\langle r_{12}^{2}\right\rangle}$. All these quantities are expected to give results of the same order. We find that the results obtained from the calculation of $\left\langle 1 / r_{12}\right\rangle^{-1}$ are closest to those determined from the pair density function. In figure 11 we show the behaviour 


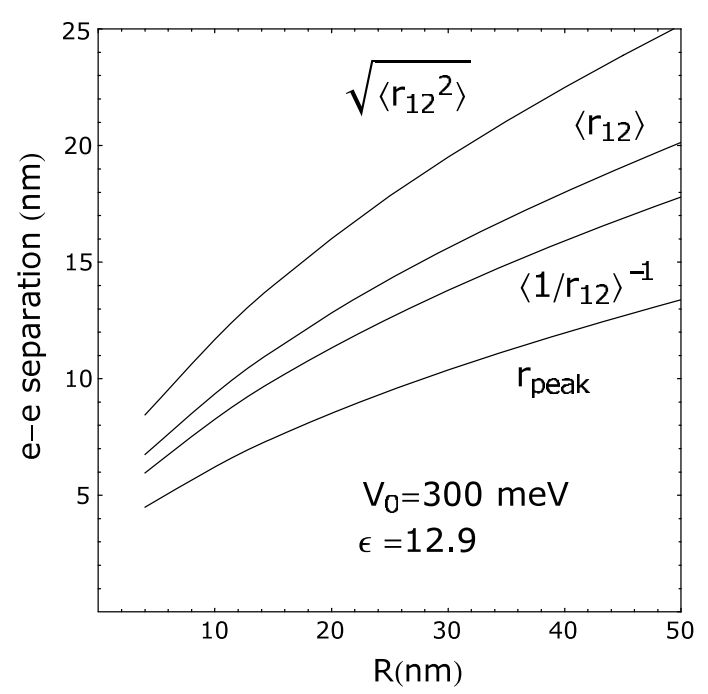

Figure 11. Variation of $\left\langle r_{12}\right\rangle,\left\langle 1 / r_{12}\right\rangle^{-1}$ and $\sqrt{\left\langle r_{12}{ }^{2}\right\rangle}$ as a function of the effective range of the confining potential, $R$, for $V_{0}=300 \mathrm{meV}$ and $\varepsilon=12.9$. The variation of $r_{\text {peak }}$ is also shown for comparison.

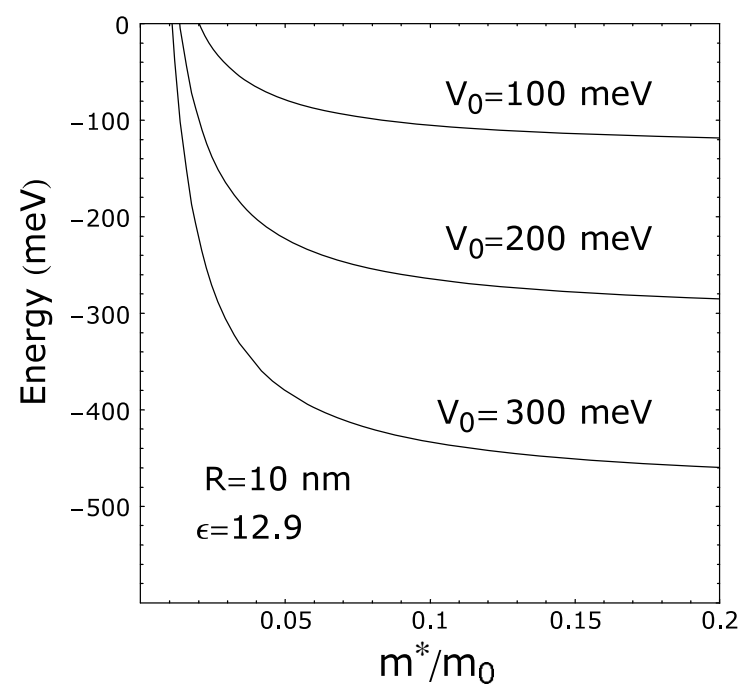

Figure 12. Variation of the two-particle bound state energy as a function of the effective mass of the electrons for $R=10 \mathrm{~nm}, \varepsilon=12.9$ and three values of $V_{0}$, namely, $V_{0}=100,200$ and $300 \mathrm{meV}$.

of these quantities as a function of $R$ and compare them with the results obtained from the pair correlation function calculation. Figure 11 shows that the pair correlation function provides the smallest values for the electron-electron separation in the bound phase, and as we have already mentioned $\left\langle 1 / r_{12}\right\rangle^{-1}$ gives values closest to the ones obtained from pair density calculation.

To study the dependence of the energy on the material parameters, we plot in figure 12 the energy as a function of the electron effective mass and in figure 13 the energy as a function of the dielectric constant. Figure 12 shows the variation of the energy as a function of the effective electron mass for three values of the confining potential. It is evident that a higher 


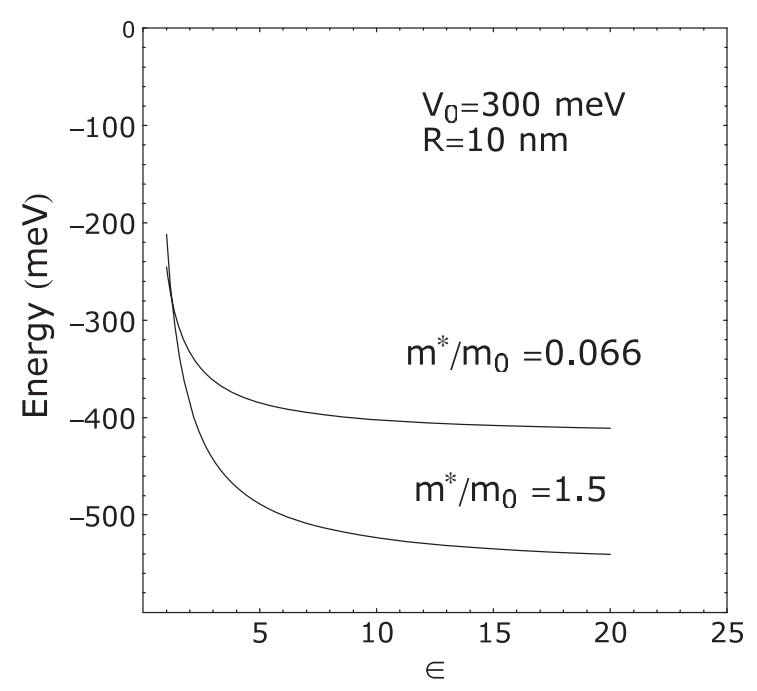

Figure 13. Variation of the two-particle bound state energy as a function of the dielectric constant for $R=10 \mathrm{~nm}, V_{0}=300 \mathrm{meV}$ and for two values of the electron mass, $m^{\star} / m_{0}=0.06$ and 1.5 .

confining potential leads to a lower energy. One can also observe that as the effective mass of the electron increases the energy decreases, which is of course understandable because an increase in the electron mass leads to a lower kinetic energy and thus helps in localization. However, as the effective mass exceeds some critical value, the energy seems to asymptotically saturate to a constant. This constant energy can be obtained from the classical minimum of the two competing potentials, namely the Gaussian potential and Coulomb interaction potential. In this limit, the total energy is more or less independent of the effective electron mass, the kinetic energy playing essentially no role. From figure 13, we observe that the energy increases with decreasing dielectric constant. This happens because as the dielectric constant decreases the effective Coulomb coupling constant increases, giving rise to stronger repulsion between the electrons, and consequently the total energy increases. We have studied the case for two effective mass values, and as expected for a higher effective mass the energy is lower. Furthermore, it is evident that as the dielectric constant exceeds a certain value (weakly depending on the effective mass of the electrons) the energy saturates to a constant independent of the dielectric constant. This is because at large values of the dielectric constant the Coulomb coupling constant becomes negligibly small and in this regime the Coulomb interaction has essentially no effect and as a result the energy becomes independent of the dielectric constant and the repulsive part in the total energy essentially comes from the kinetic energy alone. On the other hand, at the other extreme, i.e. when the dielectric constant is very small, the energy is independent of the effective mass of the electrons, kinetic energy becomes unimportant and the dominating delocalizing effect comes essentially from the Coulomb interaction only.

We have finally studied the variation of $\left\langle 1 / r_{12}\right\rangle^{-1}$ with the effective electronic mass and the dielectric constant. In figure 14 we plot $\left\langle 1 / r_{12}\right\rangle^{-1}$ as a function of the electron effective mass for $R=10, \varepsilon=12.9$ and two values of $V_{0}$, namely, $V_{0}=300$ and $30 \mathrm{meV}$, and in figure 15 we plot $\left\langle 1 / r_{12}\right\rangle^{-1}$ as a function of the dielectric constant for $R=10 \mathrm{~nm}, \varepsilon=12.9$, $V_{0}=300 \mathrm{meV}$ and two values of the effective electron mass. We observe from figure 14 that as the electron effective mass increases the electron-pair size decreases, indicating a stronger binding, and the size becomes even smaller as the depth of the confining potential is also increased. From figure 15 we find that with decreasing dielectric constant the electron-electron 


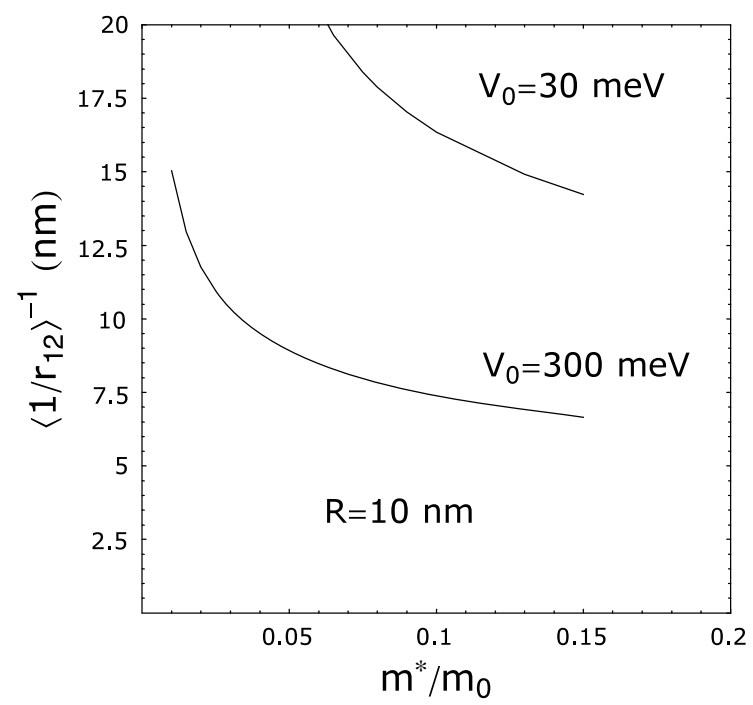

Figure 14. Variation of the electron-electron separation in the singlet bound as a function of the effective electronic mass for $R=10 \mathrm{~nm}$ and $V_{0}=30$ and $300 \mathrm{meV}$.

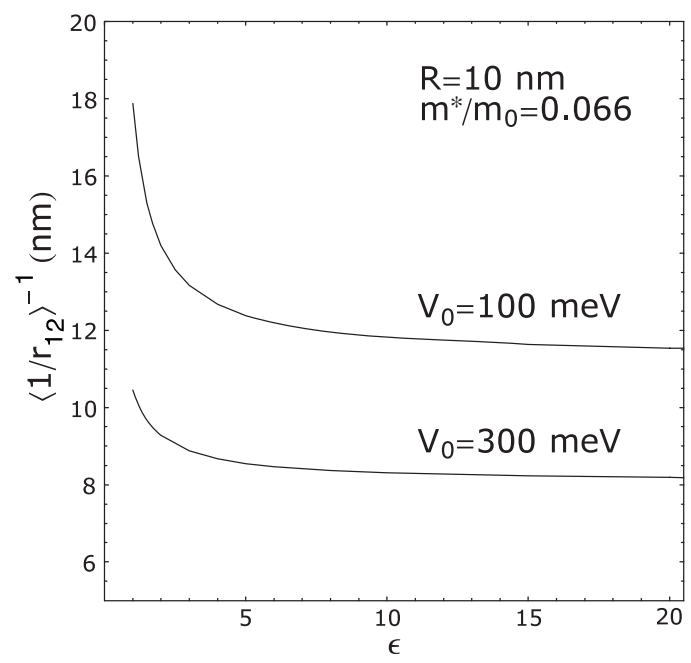

Figure 15. Variation of the two-particle bound state energy as a function of the dielectric constant for $R=10 \mathrm{~nm}, m^{\star} / m_{0}=0.06$ and $V_{0}=100$ and $300 \mathrm{meV}$.

separation increases in agreement with the behaviour observed in the case of the energy. We have shown the behaviour for two values of the potential. With a lower value of the potential, the pair size is in general larger for the same value of the dielectric constant and increase is even more rapid with decreasing dielectric constant. We also observe that there is no bound state below a certain value of the dielectric constant and this value of the critical dielectric constant increases with the decrease in the depth of the confining potential. The reason is simple. As the dielectric constant decreases, the Coulomb interaction becomes more and more repulsive, and if the effective mass is also small then the kinetic energy also tries to delocalize the electrons and in this case a small confining potential cannot provide a bound state. For 
a certain material, where the electron effective mass and dielectric constant are fixed, one can obtain the minimum potential required to have a bound state of two electrons for a certain value of the quantum dot size from the phase diagram like figure 5.

Before we end, we would however like to mention that one can easily improve our wavefunction so that it should be able to provide even better results (quantitatively) for the entire range of the confining potential. One obvious way of improving our result is to choose the following function:

$$
\psi\left(\overrightarrow{r_{1}}, \overrightarrow{r_{2}}\right)=N r_{12}^{p}\left(\mathrm{e}^{-\alpha_{1} r_{1}^{2}} \mathrm{e}^{-\alpha_{2} r_{2}{ }^{2}}+\mathrm{e}^{-\alpha_{1} r_{2}{ }^{2}} \mathrm{e}^{-\alpha_{2} r_{1}{ }^{2}}\right) \mathrm{e}^{\delta_{1} r_{12}+\delta_{2} r_{12}^{2}}
$$

where $\alpha_{1}, \alpha_{2}, \delta_{1}, \delta_{2}$ and $p$ are to be treated as variational parameters. This wavefunction is expected to improve substantially the present results for the smaller and the larger values of the confinement lengths and is also expected to give a correct phase diagram for the entire range of the depth of the confining potential. Calculation with this improved wavefunction is in progress and will be published in due course.

In conclusion, we have studied the problem of a system of two electrons in a quantum dot with Gaussian confinement for singlet pairing in three dimensions and compared the results with the corresponding ones for the parabolic potential. We have observed, in agreement with [15], that in general the parabolic potential is only a poor approximation for the Gaussian potential. We have performed a single-parameter variational calculation using a Jastrow-type correlation factor and compared our results with those of Adamowsky et al [15], who have used a large number of parameters in their wavefunction. We find that for a certain range of the confinement length, namely in the intermediate range, our single-parameter results are in very good agreement with those of Adamowsky et al. For small and large confinement lengths, of course, the results of Adamowsky et al are slightly better. But in view of the extreme simplicity of our wavefunction our results can be considered fairly good. We have also shown that there exist some critical values for the potential parameters below which one cannot have a two-electron bound state in a Gaussian quantum dot. At these values of the parameters an electron will have the tendency to tunnel through the quantum dot. We have obtained results for a few semiconductor quantum dots, and comparison shows that for the same range and depth of the Gaussian confining potential GaAs provides the widest bound phase for a singlet pair of electrons among the materials considered in our investigation. We find that in general the binding increases with increasing depth of the confining potential in all materials. We observe that the key parameters for the stability of a bound pair of electrons in a Gaussian quantum dot are essentially the electron effective mass and the material dielectric constant. We have calculated the electron-electron separation in the bound state, i.e. the electron-pair size, using different methods, and studied its variation with the material parameters. We have shown that the electron-pair size decreases with the increase of the electron mass and increases with decreasing dielectric constant. We have also indicated the way of improving on our simple wavefunction so that one can obtain even better results for the entire range of the confinement length. It would also be interesting to extend this work to include the effect of an external magnetic field and study the crossing of the singlet-triplet energy levels, because experimentally this would give the signature of the Coulomb correlation effect. Such a calculation is in progress and will be reported in due course. Another issue that is under investigation is the effect of spin-orbit interaction of Rashba type in semiconductor quantum dots considered in this paper. Though this coupling has a much smaller energy scale as compared to the Zeeman term, it does have an observable effect on the energy levels and leads to the well known fine structure splitting and energy band mixing. But more importantly, it gives rise to a mixing of up and down spin states with a nontrivial phase difference, and this might lead to some interference phenomena. 


\section{References}

[1] Kastner M A 1992 Rev. Mod. Phys. 64849

Johnson N F 1995 J. Phys.: Condens. Matter 7965

Ashoori R C 1996 Nature 379413

Woggon U 1997 Optical Properties of Semiconductor Quantum Dots (Berlin: Springer)

Jacak L, Hawrylack P and Wojs A 1998 Quantum Dots (Berlin: Sringer)

Bimberg D, Grundmann M and Ledentsov N N 1998 Quantum Dot Heterostructures (New York: Wiley)

Mukhopadhyay S and Chatterjee A 2001 Acta Phys. Pol. B 32473

Kouwenhoven L P, Austing D G and Tarucha S 2001 Rep. Prog. Phys. 64701

[2] Sikorski Ch and Merkt U 1989 Phys. Rev. Lett. 622164

Sikorski Ch and Merkt U 1990 Surf. Sci. 229282

[3] Meurer B, Heitmann D and Ploog K 1992 Phys. Rev. Lett. 681371

[4] Ashoori R C, Stormer H L, Weiner J S, Pfeiffer L N, Pearton S J, Balswin K W and West K W 1992 Phys. Rev. Lett. 683088

[5] Drexler H, Leonard D, Hansen W, Kotthaus J P and Petroff P M 1994 Phys. Rev. Lett. 732252

[6] Kohn W 1961 Phys. Rev. 1231242

[7] Peeters F M 1990 Phys. Rev. B 421486

[8] Yip S K 1991 Phys. Rev. B 431707

[9] Li Q P, Karrai K, Yip S K, Das Sarma S and Drew H D 1991 Phys. Rev. B 435151

[10] Maxym P A and Chakraborty T 1990 Phys. Rev. Lett. 65108

Gu S W and Guo K X 1993 Solid State Commun. 891023

Johnson N F and Payne M C 1991 Phys. Rev. Lett. 671157

Johnson N F and Payne F C 1992 Phys. Rev. B 453819

Egger R, Hausler W, Mak C H and Grabert H 1999 Phys. Rev. Lett. 823320

Zhu K D and Gu S W 1993 Phys. Rev. B 4712947

Zhu K D and Kobayashi T 1995 Solid State Commun. 95805

Chen C Y, Jin P W, Li W S and Lin D L 1997 Phys. Rev. 5614913

Mukhopadhyay S and Chatterjee A 1998 Phys. Lett. A 242355

Mukhopadhyay S and Chatterjee A 1995 Phys. Lett. A 204411

Mukhopadhyay S and Chatterjee A 1996 Int. J. Mod. Phys. 102781

Mukhopadhyay S and Chatterjee A 1997 Phys. Rev. B 559297

Mukhopadhyay S and Chatterjee A 1998 Phys. Rev. B 582088

Mukhopadhyay S and Chatterjee A 1998 Phys. Lett. A 240100

Mukhopadhyay S and Chatterjee A 1999 Phys. Rev. B 59 R7833

Mukhopadhyay S and Chatterjee A 1999 J. Phys.: Condens. Matter 112071

Mukhopadhyay S and Chatterjee A 2000 Int. J. Mod. Phys. B 143897

Mukhopadhyay S and Chatterjee A 2002 Int. J. Mod. Phys. B 161489

Kervan N, Altanhan T and Chatterjee A 2003 Phys. Lett. A 315280

Krishna P M and Chatterjee A 2005 Physica B 358191

Krishna P M and Chatterjee A 2005 Physica E 3064

Krishna P M, Mukhopadhyay S and Chatterjee A 2006 Solid State Commun. 138285

Krishna P M, Mukhopadhyay S and Chatterjee A 2007 Phys. Lett. A 360655

[11] Heitmann D, Bollweg K K, Gudmundsson V, Kurth T and Riege S P 1997 Physica E 1204

Miller B T, Hansen W, Manus S, Luyken R J, Lorke A, Kotthaus J P, Huant S, Mediros-Ribeiro G and Petroff P M 1997 Phys. Rev. B 566764

[12] Szafran B, Adamowsky J and Bednarek S 1999 Physica E 41

[13] Bednarek S, Szafran B and Adamowsky J 1999 Phys. Rev. B 5913036

[14] Szafran B, Adamowsky J and Bednarek S 2000 Phys. Rev. B 611971

[15] Adamowsky J, Sobkowicz M, Szafran B and Bednarek S 2000 Phys. Rev. B 624234

[16] Bessis N, Friedrich H and Wheatly C 1982 J. Phys. A: Math. Gen. 153679

Lai C S 1983 J. Phys. A: Math. Gen. 16 L181

Crandall R E 1983 J. Phys. A: Math. Gen. 16 L395

Cohen M 1984 J. Phys. A: Math. Gen. 17 L101

Chatterjee A 1985 J. Phys. A: Math. Gen. 182403

Chatterjee A 1990 Phys. Rep. 186249

[17] Brus L E 1984 J. Chem. Phys. 804403

Zhu J L et al 1997 Phys. Rev. B 5515819 
Franceschetti A et al 2000 Phys. Rev. B 622614

Yoffe A D 2001 Adv. Phys. 501 and references therein

Cantele G et al 2001 Phys. Rev. B 62125325

Bester G et al 2003 Phys. Rev. B 68073309

Parascandolo G et al 2003 Phys. Rev. B 68245318

Xie W F et al 2004 Commun. Theor. Phys. 42151

He L et al 2005 Phys. Rev. B 72195307

[18] Laughlin R B 1983 Phys. Rev. Lett. 501395

[19] Sil S, Giri A K and Chatterjee A 1991 Phys. Rev. B 4312642

[20] Mukhopadhyay S and Chatterjee A 1996 J. Phys.: Condens. Matter 84017

[21] Ciftja O and Anil Kumar A 2004 Phys. Rev. B 70205326 and references therein

Ciftja O and Faruk M G 2005 Phys. Rev. B 72205334 